\title{
Morfometria, estereologia e bioquímica hepática de ratos albinos lactentes submetidos à ingestão residual da pregabalina no leite materno
}

\author{
Morphometry, stereology and liver biochemistry tests in infant albino rats submitted to residual \\ ingestion of pregabalin in breast milk \\ Morfometría, estereología y bioquímica hepática en ratas albinas lactantes sometidas a ingestión \\ residual de pregabalina en leche materna
}

\section{Resumo}

O estudo avaliou os efeitos da pregabalina sobre o fígado de ratos lactentes, oriundos da prole de fêmeas que receberam a droga durante a lactação. Foram utilizados 24 ratos albinos lactentes com 14 e 21 dias de vida. Os animais foram divididos em grupos controle (GC) e pregabalina (GP), e estes, subdivididos, de acordo com a idade de eutanásia (GC14, GC21, GP14, GP21). As matrizes lactantes dos grupos tratados receberam por via oral, o psicofármaco pregabalina durante os 21 dias de amamentação. As matrizes dos grupos controles receberam apenas solução fisiológica. Na data da eutanásia, valores morfométricos e estereológicos foram aferidos. As amostras foram fixadas e secções de fígado foram processadas. Área e diâmetros dos núcleos de hepatócitos, área dos hepatócitos, área e diâmetro de ductos biliares e número de hepatócitos por área além de densidades de volume dos hepatócitos e do tecido intersticial foram obtidos. Os resultados foram confrontados pelo teste estatístico "U" de Mann-Whitney, para variáveis com distribuição não paramétrica, e teste "t" de Student para variáveis paramétricas. Os animais tratados apresentaram aumento de variáveis biométricas em ambas as idades estudadas. Houve aumento da área dos hepatócitos aos 14 e 21 dias de vida, e aumento do diâmetro dos núcleos dos hepatócitos dos animais eutanasiados aos 21 dias de idade. Verificou-se aumento do volume dos tecidos hepáticos no grupo de 14 dias de vida. As enzimas hepáticas não sofreram alterações. A pregabalina promoveu hipertrofia de hepatócitos (Megalocitose) sem comprometimento da atividade funcional das células.

Palavras-chave: Biometria; Fígado; Psicofármaco; Pregabalina. 


\begin{abstract}
This study has evaluated the effects of pregabalin on the liver of lactating rats, derived from the offspring of mothers who received the drug during lactation. Twenty-four albino lactating rats with 14 and 21 days of life were used. They were divided into control (CG) and pregabalin (PG) groups, and these were subdivided according to the age at euthanasia (CG14, CG21, PG14, PG21). The lactating mothers of the treatment group received, orally, the psychotropic drug pregabalin during the 21 days of nursing. The ones of the control group received only saline solution. On the date of euthanasia, morphometric and stereological values were measured. The samples were fixed and liver sections were processed. Area and diameter of hepatocyte nuclei; area of hepatocytes; area and diameter of bile ducts; and the number of hepatocytes per area; as well as volume densities of hepatocytes, and interstitial tissue were obtained. The results were compared by the Mann-Whitney U statistical test, for variables with non-parametric distribution, and Student's t-test for parametric variables. Animals in the treatment group showed an increase in biometric variables at both ages studied. There was an increase in the area of hepatocytes at 14 and 21 days of life, and an increase in the diameter of the hepatocyte nuclei of euthanized animals at 21 days of age. There was an increase in the volume of liver tissues in the 14-day-old group. Liver enzymes were unchanged. Pregabalin promoted hepatocyte hypertrophy (Megalocytosis) without compromising the functional activity of the cells.
\end{abstract}

Keywords: Liver; Pregabalin; Psychopharmaceutical; Stereology.

\title{
Resumen
}

El estudio evaluó los efectos de la pregabalina en el hígado de ratas lactantes de la descendencia de hembras que recibieron el fármaco durante la lactancia. Se utilizaron veinticuatro ratas lactantes albinas con 14 y 21 días de vida. Los animales se dividieron en grupos de control (CG) y pregabalina (GP), y estos se subdividieron según la edad de eutanasia (GC14, GC21, GP14, GP21). Las madres lactantes de los grupos tratados recibieron, por vía oral, el fármaco psicotrópico pregabalina durante los 21 días de lactancia. Las cerdas de los grupos de control recibieron solo solución salina. En la fecha de la eutanasia se midieron los valores morfométricos y estereológicos. Las muestras se fijaron y se procesaron secciones de hígado. Se obtuvieron el área y diámetro de los núcleos de los hepatocitos, el área de los hepatocitos, el área y el diámetro de los conductos biliares y el número de hepatocitos por área, así como las densidades de volumen de los hepatocitos y el tejido intersticial. Los resultados se compararon mediante la prueba estadística " $U$ " de Mann-Whitney, para variables con distribución no paramétrica, y la prueba "t" de Student para variables paramétricas. Los animales tratados mostraron un aumento de las variables biométricas en las dos edades estudiadas. Hubo un aumento en el área de los hepatocitos a los 14 y 21 días de vida, y un aumento en el diámetro de los núcleos de los hepatocitos de los animales sacrificados a los 21 días de edad. Hubo un aumento en el volumen de tejidos hepáticos en el grupo de 14 días. Las enzimas hepáticas no cambiaron. La pregabalina promovió la hipertrofia de los hepatocitos (megalocitosis) sin comprometer la actividad funcional de las células.

Palabras clave: Estereologí; Hígado; Psicofármaco; Pregabalina.

\section{Introdução}

A pregabalina é um análogo do neurotransmissor inibitório ácido gama-aminobutírico (GABA), age atenuando a liberação de neurotransmissores excitatórios e bloqueando o desenvolvimento da hiperalgesia e sensibilização central (Martins, Panício, Dantas \& Gomes, 2014).

Em comparação com outros efeitos colaterais, como fadiga, ganho de peso e tremor, o efeito sobre a função hepática é considerado mais problemático e importante razão de não aderência ao tratamento (Cano-Paniagua et al. 2019; Cardia et al. 2021; Machado, 2021; Silveira et al. 2019).

A proposta do projeto é de quantificar os efeitos da pregabalina sobre o parênquima hepático, subsidiando à posterior definição de posologias terapêuticas mais seguras, que não comprometam a função desse órgão e a qualidade de vida dos pacientes.

\section{Metodologia}

Esta pesquisa foi previamente submetida ao Comitê de Ética para Uso de Animais (CEUA) do Centro de Saúde e Tecnologia Rural (CSTR) da Universidade Federal de Campina Grande (UFCG), Campus de Patos, Paraíba, Brasil, tendo sido aprovada sob protocolo CEUA/CSTR N 030/2020. 


\subsection{Local do Trabalho}

O experimento foi realizado no Biotério Animal, Laboratórios de Patologia Animal, Laboratório de Patologia Clínica do Hospital Veterinário e no Laboratório de Morfofisiologia do Centro de Saúde e Tecnologia Rural da Universidade Federal de Campina Grande, Paraíba, Brasil.

\subsection{Formação dos grupos controles e tratados}

Os grupos foram formados com 24 ratos albinos (Rattus norvegicus albinus), pertencentes à linhagem Wistar. Durante o período de amamentação, que transcorreu pelo prazo de 14 e 21 dias, os ratos ficaram em gaiolas plásticas com água e alimentação ad Libitum. Os animais foram divididos em dois grupos principais: o grupo controle (GC) com 12 animais, e um grupo tratado (GP), também com 12 animais.

As matrizes do grupo tratado receberam $60 \mathrm{mg} / \mathrm{kg} / \mathrm{dia}$ de pregabalina por via oral (gavage) durante o período de amamentação. As matrizes do grupo controle receberam solução salina diariamente por via oral. Os grupos principais foram subdivididos em quatro subgrupos de seis animais cada, de acordo com as idades em que os animais foram eutanasiados (14 e 21 dias de vida), compondo os subgrupos GC14, GC21, GP14 e GP21.

As idades de eutanásia foram escolhidas com base no tempo médio e máximo de amamentação (14 e 21 dias). Nas idades especificadas, os animais foram anestesiados com Tiopental Sódico ( $89 \mathrm{mg} / \mathrm{kg}$ de peso corpóreo).

\subsection{Coleta e processamento das amostras}

Nas idades especificadas, os ratos foram pesados e submetidos à eutanásia. Amostras de sangue foram colhidas, centrifugadas e conduzidas ao Laboratório de Patologia Clínica do Hospital Veterinário para determinação das dosagens de AST, ALT e FA.

O fígado foi removido e pesado em balança semianalítica de precisão BG 1000 GEHAKA®. As amostras foram prefixadas por imersão em formaldeído, e após 30 minutos, hemisseccionadas. As hemissecções retornaram à solução fixadora permanecendo por 24 horas. Após a fixação, as amostras foram processadas no Laboratório de Patologia Animal (LPA) do Hospital Veterinário Universitário Prof. Ivon Macêdo Tabosa (HVU) do CSTR, da UFCG, Campus de Patos, Paraíba; em processo semelhante ao realizado por Michalany (1998).

Foram obtidas secções de $5 \mu \mathrm{m}$ de fígado por meio do micrótomo manual LEICA RM2125 RT e navalhas descartáveis Easy Path DURAEDGE. Três secções não simultâneas do fígado de cada animal foram obtidas e coradas pela Hematoxilina e Eosina, possibilitando a análise morfométrica, estereológica e histológica de cada órgão. Os cortes histológicos foram analisados em microscópio de bancada da marca Olympus BX41, utilizando a objetiva de 20x e 40x.

\subsection{Biometria, Morfometria e Estereologia}

A biometria incluiu o peso corpóreo dos animais, o peso absoluto e relativo do fígado. O peso relativo de cada órgão foi calculado mediante a fórmula (peso do órgão/100g de peso vivo) descrita por Brilhante (1999). O volume de cada órgão foi aferido pelo método de Scherle (1970). O valor deve ser registrado e descontado o peso do fio agulhado para obter o volume do órgão (Mandarim-de-Lacerda, 1995). A gravidade específica é a razão entre a densidade do órgão avaliado e a densidade da substância de referência (água) (Russel \& França, 1995). Desta forma, o volume total foi obtido a partir da seguinte fórmula:

\section{VOLUME=Densidade X Massa}

Para cada corte foi determinado o número de hepatócitos por área em micrômetro quadrado $(\mu \mathrm{m} 2)$. Em 100 hepatócitos por corte (300 por animal), foram mensurados a área $(\mu \mathrm{m} 2)$ dos hepatócitos e núcleos de hepatócitos. Também 
foram aferidos os diâmetros $(\mu \mathrm{m})$ e a áreas $(\mu \mathrm{m} 2)$ dos ductos biliares em 10 estruturas por corte (30 por animal), quando cortadas transversalmente. Todas as aferições foram efetuadas de forma aleatória e uniformemente variada através da varredura sistemática das secções em ziguezague, após a obtenção de fotomicrografias por meio do Software IMAGE PRO EXPRESS 6.0 em computador acoplado em microscópio binocular de luz OLYMPUS BX40 no aumento de 40x.

A estereologia incluiu a densidade de volume ( $\mathrm{Vv}$ ) do parênquima e do tecido intersticial. As variáveis estereológicas de densidade de volume ( $\mathrm{Vv}$ ) foram obtidas por meio da contagem dos pontos por alocação sistemática e aleatória das imagens obtidas através do software IMAGE-PRO EXPRESS 6.0 e posteriormente empregada no software IMAGEJ para a contagem dos pontos totalizando 960 pontos por órgão (Gunderson \& Dygert, 1988; Mandarim-de-Lacerda, 1995).

A densidade dos tecidos intersticial e do parênquima $\mathrm{em}^{3} \mathrm{~m}^{3}$ foi calculada a partir do percentual obtido na densidade de volume (Vv) de cada tecido segundo Miraglia e Hayashi (1993). Os volumes do parênquima e do tecido intersticial médios por animal também foram obtidos através do cálculo com os dados obtidos para o volume total dos órgãos.

\subsection{Análise Estatística}

Os resultados foram submetidos ao teste de normalidade, os dados com distribuição não paramétrica foram submetidos ao teste "U" de Mann-Whitney. Os dados com distribuição paramétrica foram confrontados pelo teste " $\mathrm{t}$ " de Student. Ambos os testes consideraram grau de significância de 5\%.

\section{Resultados}

Os animais dos grupos GP e GC foram observados diariamente e nenhum animal veio à óbito durante o experimento. Os lactentes permaneceram clinicamente hígidos e com características comportamentais dentro do padrão de normalidade para a espécie.

As variáveis foram apresentadas por médias aritméticas dos grupos GP e GC. Os dados biométricos incluíram as médias do peso corpóreo dos animais, peso absoluto e relativo do fígado e volume do fígado dos grupos GP e GC (Tabela 1).

Tabela 1 - Médias de Peso Corpóreo (PC), Peso Absoluto do Fígado (PF), Peso Relativo do Fígado (PRF) e Volume do Fígado (VF) dos ratos albinos dos grupos controle (GC) e pregabalina (GP), eutanasiados aos 14 e 21 dias de vida e seus respectivos desvios padrões (DP).

\section{GRUPOS}

\begin{tabular}{lcccccccc}
\hline & $\mathrm{GC}_{14}$ & $\mathbf{D P}( \pm)$ & $\mathrm{GP}_{14}$ & $\mathbf{D P}( \pm)$ & $\mathrm{GC}_{21}$ & $\mathbf{D P}( \pm)$ & $\mathrm{GP}_{21}$ & $\mathbf{D P}( \pm)$ \\
\hline $\mathbf{P C}(\mathbf{g})$ & $27 \mathrm{a}$ & 2,75 & $32 \mathrm{~b}$ & 3,20 & $42,2 \mathrm{c}$ & 6,04 & $65 \mathrm{~d}$ & 4,36 \\
$\mathbf{P F}(\mathbf{g})$ & $0,81 \mathrm{a}$ & 0,09 & $1,17 \mathrm{~b}$ & 0,08 & $1,65 \mathrm{c}$ & 0,31 & $2,70 \mathrm{~d}$ & 0,40 \\
$\mathbf{P R F}(\mathbf{g})$ & $3,02 \mathrm{a}$ & 0,14 & $3,68 \mathrm{~b}$ & 0,44 & $3,86^{*} \mathrm{c}$ & 0,20 & $4,17^{*} \mathrm{c}$ & 0,63 \\
$\mathbf{V F}\left(\mathbf{m m}^{3}\right)$ & $0,63 \mathrm{a}$ & 0,08 & $1,06 \mathrm{~b}$ & 0,09 & $1,45 \mathrm{c}$ & 0,32 & $2,48 \mathrm{~d}$ & 0,39 \\
& & & & & & & & \\
\hline
\end{tabular}

Observação: Letras diferentes-Médias diferiram entre si pelo teste " $t$ " de Student ( $\mathrm{p} \leq 0,05)$. Letras iguais-Médias não diferiram entre si pelo teste " $t$ " de Student $(\mathrm{p}>0,05)$. Letras diferentes com "*” - Médias diferiram entre si pelo teste "U" de Mann-Whitney ( $\leq 0,05)$. Letras iguais com “*” - Médias não diferiram entre si pelo teste "U” de Mann-Whitney (p>0,05). Fonte: Autores. 
O peso corpóreo dos grupos experimentais de 14 dias mostrou-se diferente quando comparados entre si $(\mathrm{p}=0,0131)$, houve um incremento de $15,6 \%$ no peso corpóreo dos animais tratados com psicofármaco pregabalina (GP14). O aumento de peso também foi observado no grupo GP21 quando comparado ao grupo controle de mesma idade (p=0,0001), todavia nessa idade o incremento de peso $(35,07 \%)$ foi percentualmente maior que nos grupos experimentais de 14 dias.

$\mathrm{O}$ peso do fígado mostrou-se aumentado nos grupos experimentais de ambas as idades estudadas (GP14 p=0,002; e GP21 p=0,0006), todavia o incremento de peso da glândula no grupo GP21 (34,30\%) foi maior que no grupo GP14 (26,27\%) quando comparados aos respectivos grupos controles de mesma idade.

$\mathrm{O}$ peso relativo do fígado encontrou-se aumentado no grupo tratado com pregabalina GP14 (16,86\%) quando comparado ao grupo controle de mesma idade ( $\mathrm{p}=0,0125)$, contudo não se constatou diferença (6,96\%) nos grupos experimentais de 21 dias $(\mathrm{p}=0,152)$.

$\mathrm{O}$ volume do fígado encontrou-se aumentado nos grupos experimentais tratados com o psicofármaco em ambas as idades (GP14 p=0,0001; GP21 p=0,0009), as variações entre os grupos tratados e seus respectivos controles foram igualmente significantes tanto no período médio quanto final da lactação (GP14 30,47\%; GP21 34,93\%).

As variáveis morfométricas dos grupos GP e GC incluíram as médias da área do hepatócito, área do núcleo do hepatócito, diâmetro do hepatócito, diâmetro do núcleo do hepatócito, área do ducto biliar, diâmetro do ducto biliar e número de hepatócitos por área (Tabela 2).

Tabela 2 - Médias da Área do Hepatócito (AH), Área do Núcleo do Hepatócito (ANH), Diâmetro do Hepatócito (DH), Diâmetro do Núcleo do Hepatócito (DNH), Área do Ducto Biliar (ADB), Diâmetro do Ducto Biliar (DDB) e Número de Hepatócitos por Área (NHPA) dos ratos albinos dos grupos controle e pregabalina, eutanasiados aos 14 e 21 dias de vida e seus respectivos desvios padrões (DP).

\section{GRUPOS}

\begin{tabular}{|c|c|c|c|c|c|c|c|c|}
\hline & GC14 & DP $( \pm)$ & GP14 & DP $( \pm)$ & GC21 & DP $( \pm)$ & GP21 & DP ( $( \pm)$ \\
\hline $\mathbf{A H}\left(\mu \mathbf{m}^{2}\right)$ & $133,89 a$ & 3,56 & $161,45 b$ & 3,45 & $187,77 \mathrm{c}$ & 2,04 & $226,60 d$ & 2,44 \\
\hline $\mathrm{ANH}\left(\mu \mathrm{m}^{2}\right)$ & $34,22 \mathrm{a}$ & 2,46 & $32,00 \mathrm{a}$ & 1,79 & $37,12 \mathrm{c}$ & 2,41 & $39,91 d$ & 1,36 \\
\hline DNH $(\mu \mathrm{m})$ & $6,75 a$ & 0,31 & $6,51 \mathrm{a}$ & 0,37 & $7,25 \mathrm{c}$ & 0,34 & $7,80 \mathrm{~d}$ & 0,12 \\
\hline NHPA (und) & $39,32 \mathrm{a}$ & 1,75 & $37,79 \mathrm{a}$ & 1,17 & $38,26 \mathrm{c}$ & 0,98 & $37,88 \mathrm{c}$ & 0,89 \\
\hline $\operatorname{ADB}\left(\mu \mathrm{m}^{2}\right)$ & $241,39 a$ & 23,86 & $251,78 \mathrm{a}$ & 17,92 & $274,32 \mathrm{c}$ & 24,47 & $289,91 \mathrm{c}$ & 10,05 \\
\hline DDB $(\mu \mathrm{m})$ & $19,43 a$ & 0,9 & $20,84 a$ & 2,75 & $20,62 c$ & 1,55 & $21,34 \mathrm{c}$ & 4,25 \\
\hline
\end{tabular}

Observação: Letras diferentes-Médias diferiram entre si pelo teste " $t$ " de Student ( $p \leq 0,05)$. Letras iguais-Médias não diferiram entre si pelo teste "t" de Student ( $p>0,05)$. Fonte: Autores.

A área do hepatócito apresentou tendência de aumento em ambas as idades, sendo o acréscimo de 17,07\% aos 14 dias de vida e aos 21 dias aumento de 17,13\%. Os aumentos mostraram-se significantes em ambas as idades $(\mathrm{p}<0,0001)$.

A área do núcleo do hepatócito dos animais eutanasiados aos 14 dias de vida não apresentou diferença $(6,48 \%)$ quando comparado ao grupo controle de mesma idade (p=0,0525). Entretanto, aos 21 dias houve acréscimo de 6,99\% na área do núcleo da célula nos animais do grupo tratado com o psicofármaco (p=0,0168). 
O diâmetro do núcleo do hepatócito não apresentou diferença ( $\mathrm{p}=0,1275)$ aos 14 dias de vida, havendo oscilação de $3,55 \%$. No grupo tratado de 21 dias houve aumento significativo de 7,05\% quando comparado ao grupo controle da mesma idade $(\mathrm{p}=0,0052)$.

Neste estudo, o citoplasma de muitas células megalocíticas continham grandes áreas acidófilas focais e pequenas áreas lipídicas (vacúolos citoplasmáticos).

Não houve diferença quanto ao número de hepatócitos por área aos $14(3,89 \%, \mathrm{p}=0,0533)$ e aos $21(0,99 \%, \mathrm{p}=0,2523)$ dias de vida.

A área do ducto biliar não diferiu no grupo tratado com pregabalina aos 14 dias de vida $(4,12 \%, \mathrm{p}=0,2069)$, os animais eutanasiados aos 21 dias de vida também não apresentaram diferença nesta variável $(5,17 \%, \mathrm{p}=0,0896)$.

Não se observou diferença no diâmetro do ducto biliar nos animais eutanasiados aos 14 dias $(6,76 \%, p=0,1388)$ e aos 21 dias de vida $(3,37 \%, \mathrm{p}=0,3556)$.

O parênquima hepático dos animais dos grupos controle de 14 (Figura 1) e 21 (Figura 2) dias exibia características microscópicas compatíveis com o padrão de normalidade, todavia, algumas secções apresentaram raros vacúolos intracitoplasmáticos em hepatócitos.

Figura 1: Fotomicrografia do fígado de ratos albinos do grupo controle e eutanasiados aos 14 dias de vida (GC14). Coloração HE. 40x. Evidencia-se placas de hepatócitos (H), sinusóides hepáticos ( $\mathrm{SH})$ e células de Kupffer (K) em condições de normalidade e veia centrolobular (VCL).

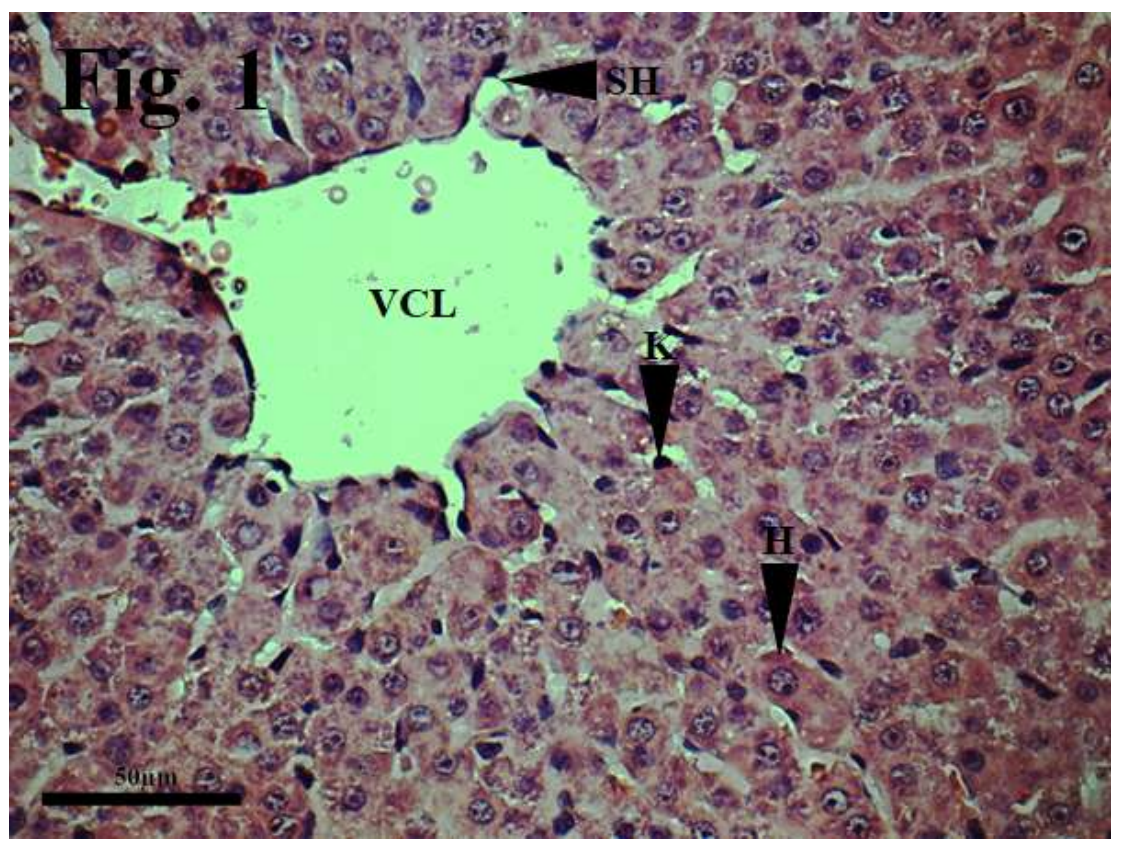

Fonte: Autores. 
Figura 2: Fotomicrografia do fígado de ratos albinos do grupo controle e eutanasiados aos 21 dias de vida (GC21). Coloração HE. 40x. Evidencia-se placas de hepatócitos $(\mathrm{H})$, sinusóides hepáticos $(\mathrm{SH})$ e células de Kupffer $(\mathrm{K})$ em condições de normalidade e veia centrolobular (VCL).

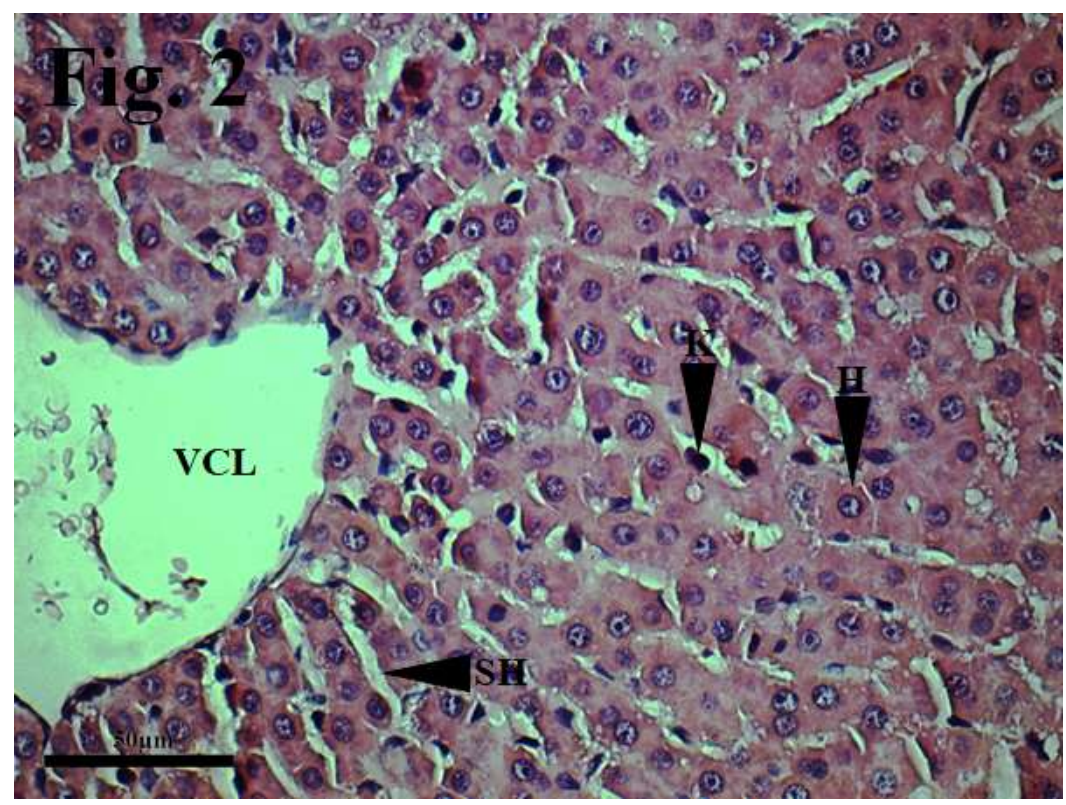

Fonte: Autores.

O parênquima hepático dos animais dos grupos tratados com pregabalina e eutanasiados aos 14 dias de vida (Figura 3) apresentaram vários vacúolos intracitoplasmáticos em hepatócitos, acompanhado por grau moderado de desorganização e degradação do parênquima hepático, exibindo a espaços regulares infiltrados inflamatórios.

Figura 3: Fotomicrografia do fígado de ratos albinos do grupo tratado com pregabalina e eutanasiados aos 14 dias de vida (GP14). Coloração HE. 40x. Evidencia-se desorganização, degradação e vacuolizações no parênquima hepático. Placas de hepatócitos (H), sinusóides hepáticos (SH), células de Kupffer (K), infiltrado inflamatório (IF), vacúolo (V) e veia centrolobular (VCL).

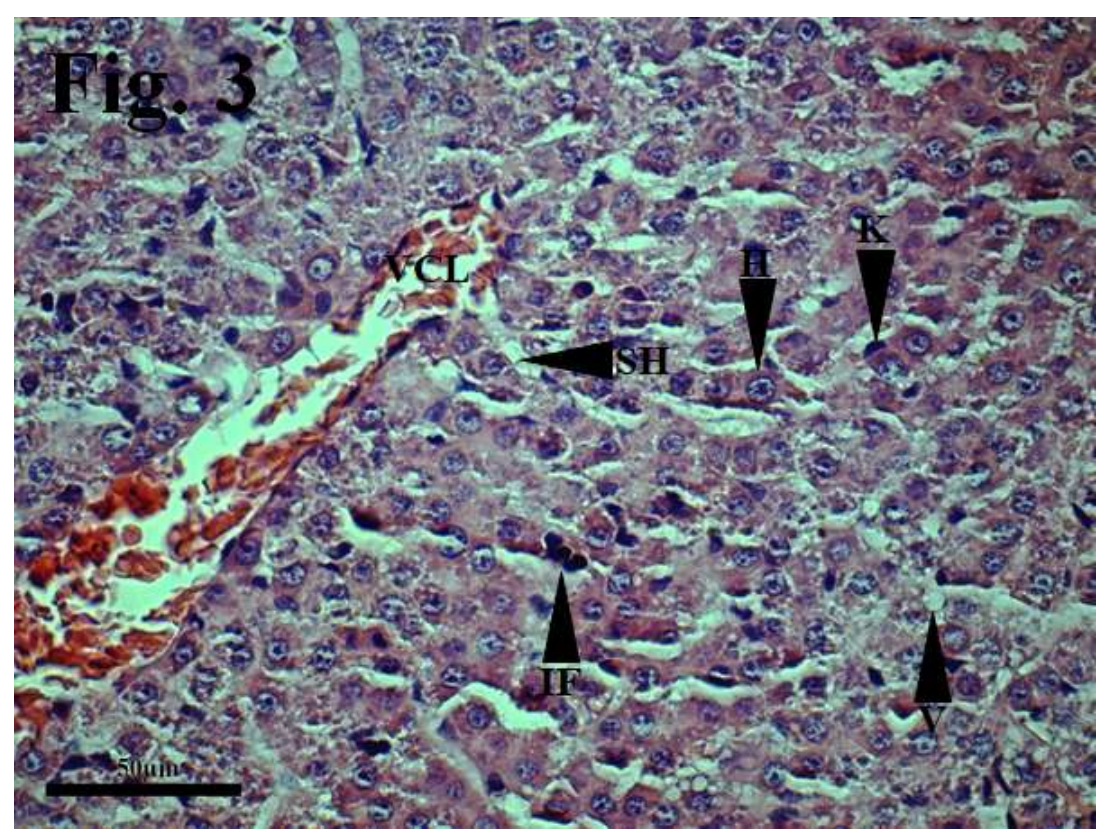

Fonte: Autores. 
O tecido hepático dos animais tratados e eutanasiados aos 21 dias de vida (Figura 4) expressou diversos vacúolos intracitoplasmáticos em hepatócitos, células hepáticas em processo avançando de divisão (células binucleadas). Aos 21 dias de tratamento a desorganização e degradação do parênquima hepático mostrou-se mais avançada que no grupo tratado de 14 dias de vida.

Figura 4: Fotomicrografia do fígado de ratos albinos do grupo tratado com pregabalina e eutanasiados aos 21 dias de vida (GP21). Coloração HE. 40x. Evidencia-se desorganização, degradação e vacuolizações numerosas no parênquima hepático. Placas de hepatócitos $(\mathrm{H})$, sinusóides hepáticos $(\mathrm{SH})$, células de Kupffer $(\mathrm{K})$, célula binucleada (CB), vacúolo (V) e veia centrolobular (VCL).

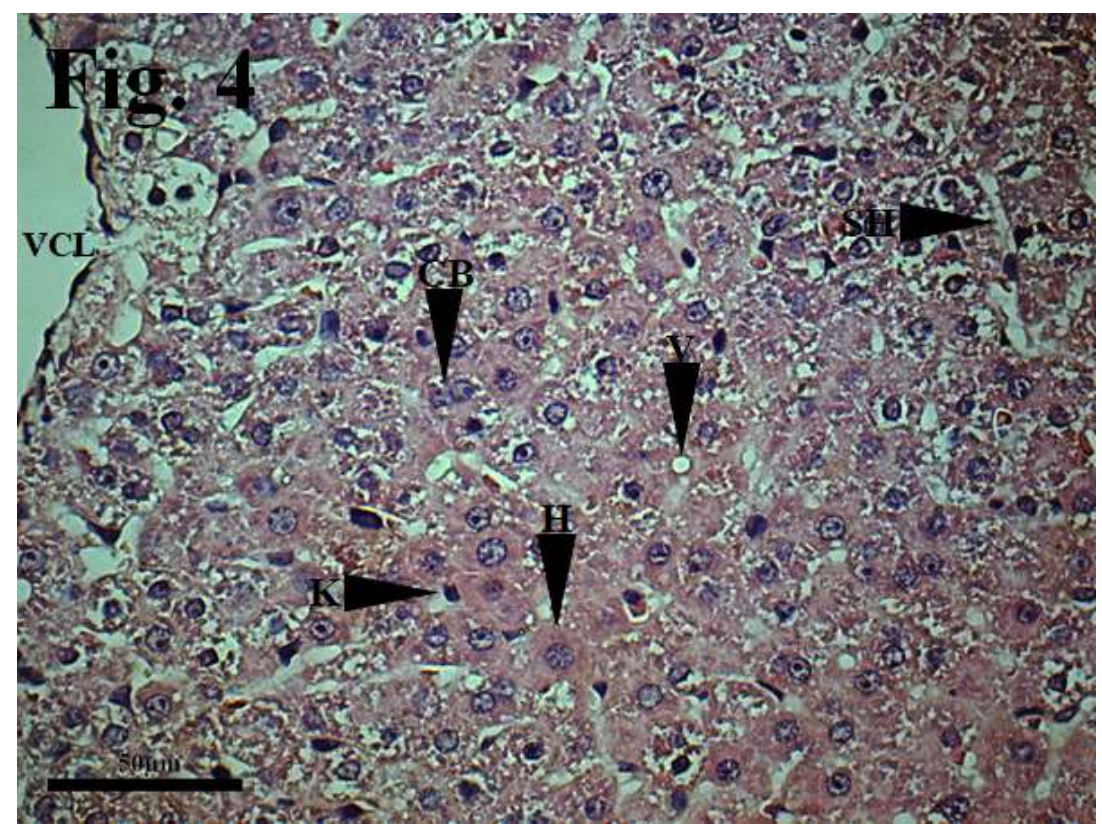

Fonte: Autores.

As variáveis estereológicas dos grupos GP e GC incluíram as médias de densidade de volume do tecido intersticial, densidade de volume do parênquima hepático, volume do tecido intersticial e volume do parênquima hepático (Tabela 3).

Tabela 3 - Médias da Densidade de Volume do Tecido Intersticial (VvTI), Densidade de Volume do Parênquima (VvP), Volume do Tecido Intersticial (VTI) e Volume do Parênquima (VP) dos ratos albinos dos grupos controle e pregabalina, eutanasiados aos 14 e 21 dias de vida e seus respectivos desvios padrões (DP).

\section{GRUPOS}

\begin{tabular}{|c|c|c|c|c|c|c|c|c|}
\hline & GC14 & $\mathbf{D P}( \pm)$ & GP14 & $\mathbf{D P}( \pm)$ & GC21 & $\mathbf{D P}( \pm)$ & GP21 & $\mathbf{D P}( \pm)$ \\
\hline VvTI (\%) & $15,88 \mathrm{a}$ & 1,14 & $13,59 \mathrm{~b}$ & 0,38 & $14,08 \mathrm{c}$ & 0,93 & $14,45 \mathrm{c}$ & 0,68 \\
\hline VvP (\%) & $84,12 \mathrm{a}$ & 1,14 & $86,41 \mathrm{~b}$ & 0,38 & $85,91 \mathrm{c}$ & 0,93 & $85,55 \mathrm{c}$ & 0,67 \\
\hline $\operatorname{VTI}\left(\mathrm{mm}^{3}\right)$ & $116,54 \mathrm{a}$ & 19,43 & $143,67 \mathrm{~b}$ & 13,89 & $227,61 \mathrm{c}$ & 43,44 & $360,47 \mathrm{~d}$ & 55,75 \\
\hline $\mathbf{V P}\left(\mathbf{m m}^{\mathbf{3}}\right)$ & $613,55 \mathrm{a}$ & 64,32 & $912,99 \mathrm{~b}$ & 80,91 & $1392,37 \mathrm{c}$ & 287,63 & $2137,86 \mathrm{~d}$ & 338,67 \\
\hline
\end{tabular}

Observação: Letras diferentes-Médias diferiram entre si pelo teste " $\mathrm{t}$ " de Student $(\mathrm{p} \leq 0,05)$. Letras iguais-Médias não diferiram entre si pelo teste "t" de Student ( $>0,05)$. Fonte: Autores. 
A densidade de volume do tecido intersticial apresentou redução de $14,42 \%$ no grupo tratado de 14 dias $(\mathrm{p}=0,0017)$ quando comparado ao respectivo grupo controle. O volume do tecido intersticial apresentou acréscimo de $18,88 \%$ no grupo tratado com pregabalina aos 14 dias $(\mathrm{p}=0,0097)$.

A densidade de volume do parênquima aumentou em 2,65\% aos 14 dias de vida ( $\mathrm{p}=0,0017)$. O volume do parênquima nos animais de 14 dias de vida também se mostrou diferente quando comparados os grupos controle e tratado ( $p=0,0001$ ), havendo acréscimo de 32,79\% na média dos animais do grupo GP.

Aos 21 dias de vida não houve diferença $(2,56 \%, \mathrm{p}=0,2283)$ na densidade de volume do tecido intersticial. Porém, nesta idade houve aumento de $36,85 \%$ na média do volume do tecido intersticial absoluto dos animais tratados com a droga $(\mathrm{p}=0,0005)$.

Nos animais eutanasiados aos 21 dias não houve diferença $(0,41 \%, \mathrm{p}=0,2283)$ na densidade de volume do parênquima. Todavia, houve aumento de $34,87 \%(\mathrm{p}=0,0011)$ no volume do parênquima do grupo GP.

As variáveis bioquímicas dos grupos GP e GC incluíram as médias de alanina aminotransferase, aspartato aminotransferase e fosfatase alcalina (Tabela 3).

Tabela 4 - Médias das enzimas alanina aminotransferase (ALT/TGP), aspartato aminotransferase (AST/TGO), fosfatase alcalina (FAL) dos ratos albinos dos grupos controle e pregabalina, eutanasiados aos 14 e 21 dias de vida e seus respectivos desvios padrões (DP).

\section{GRUPOS}

\begin{tabular}{lcccccccc}
\hline & GC14 & DP $( \pm)$ & GP14 & DP $( \pm)$ & GC21 & DP $( \pm)$ & GP21 & DP $( \pm)$ \\
\hline ALT/TGP (U/L) & $9,5 \mathrm{a}$ & 2,16 & $7,66 \mathrm{a}$ & 1,96 & $17,33 * \mathrm{c}$ & 42,26 & $17,5 * \mathrm{c}$ & 47,32 \\
AST/TGO (U/L) & $146 \mathrm{a}$ & 18,92 & $178,33 \mathrm{a}$ & 57,23 & $140,5 \mathrm{c}$ & 21,32 & $123,5 \mathrm{c}$ & 14,18 \\
FAL (U/L) & $46,14 \mathrm{a}$ & 22,05 & $32,42 \mathrm{a}$ & 30,07 & $72,28 \mathrm{c}$ & 66,43 & $97,42 \mathrm{c}$ & 61,63 \\
\hline
\end{tabular}

Observação: Letras diferentes-Médias diferiram entre si pelo teste " $t$ " de Student $(\mathrm{p} \leq 0,05)$. Letras iguais-Médias não diferiram entre si pelo teste " $\mathrm{t}$ " de Student $(\mathrm{p}>0,05)$. Letras diferentes com “*” -Médias diferiram entre si pelo teste " $U$ " de Mann-Whitney ( $\mathrm{p} \leq 0,05)$. Letras iguais com "*”" -Médias não diferiram entre si pelo teste " $U$ " de Mann-Whitney ( $p>0,05)$. Fonte: Autores.

A alanina aminotransferase não diferiu no grupo de 14 dias $(19,36 \%, \mathrm{p}=0,0779)$. No grupo tratado de 21 dias também não houve diferença nos níveis séricos da enzima $(1,14 \%, \mathrm{p}=0,2117)$. A enzima aspartato aminotransferase também não diferiu nos grupos tratados de 14 dias $(18,12 \%, \mathrm{p}=0,1184)$ e de 21 dias $(12,09 \%, \mathrm{p}=0,0675)$ quando comparados aos seus respectivos grupos controles. O mesmo ocorreu com a fosfatase alcalina, que não apresentou diferença nos grupos de 14 dias $(29,73 \%$, $\mathrm{p}=0,0123)$ e de 21 dias $(25,80 \%, \mathrm{p}=0,2645)$.

\section{Discussão}

O aumento do peso corpóreo dos pacientes tratados com psicofármacos já foi descrito em outros estudos (Cabrera et al., 2012; Gaspari et al., 2010; Jallon \& Picard, 2001; Ness-Abramof \& Apovian, 2005). Conforme observado em outras pesquisas, há uma tendência de aumento de pesos progressivamente maiores à medida em que o uso da droga vai se tornando crônico (Arroyo et al., 2004; Ben-Menachem, 2007; Beydoun et al., 2005; DeToledo, Toledo, DeCerce \& Ramsay, 1997; Siddall et al., 2006).

$\mathrm{O}$ aumento do peso do fígado evidencia a influência maior da droga sobre o desenvolvimento corpóreo na cronicidade. O tamanho de vários órgãos é diretamente proporcional ao desenvolvimento corpóreo (Medeiros et al. 2021; 
Rodrigues et al. 2021), portanto ao constatar-se o aumento do peso corpóreo dos animais tratados com pregabalina, espera-se que haja um aumento equivalente do peso do fígado. Entretanto, esse aumento foi percentualmente maior no fígado do que no peso corpóreo como um todo, sobretudo nos animais do grupo experimental de 14 dias. É possível que o psicofármaco tenha potencializado a diferença no peso do fígado na fase aguda do tratamento.

Segundo González-Escalada (2005) a pregabalina não é metabolizada no fígado, contudo o hepatócito é uma célula funcionalmente dinâmica interagindo direta ou indiretamente com várias moléculas existentes no plasma sanguíneo.

O aumento do peso absoluto do fígado acompanhado por aumento do peso relativo, é forte indicativo de que os efeitos da pregabalina resultaram em um aumento dos componentes teciduais hepáticos.

Frequentemente, o peso do fígado está diretamente ligado ao volume hepático, nestes casos, o aumento do peso implica necessariamente no aumento do volume, contanto que não haja alterações na densidade tecidual do órgão.

Embora a pregabalina não seja metabolizada no fígado (Gonzalez-Escalada, 2005), o princípio ativo da droga permanece circulante, interagindo com células e outros componentes do tecido, ou seja, a inexistência de transformação metabólica de uma substância pelo hepatócito não garante ausência de hepatotoxicidade de uma droga (Einarsdottir \& Björnsson, 2008). Ademais, a pregabalina é análoga ao receptor GABA, a administração de substâncias análogas ao GABA pode resultar no aumento da produção de amônia como resultado da atividade cíclica dos neurotransmissores (Kvamme, Torgner, \& Roberg, 2001).

O aumento dos níveis séricos de amônia pode resultar em sobrecarga metabólica dos hepatócitos. O tamanho dos hepatócitos pode variar em decorrência alterações metabólicas ou no aparecimento de lesões degenerativas (Seawright et al., 1991).

A hipertrofia do hepatócito já foi descrita em outros estudos como medida compensatória a um aumento de demanda metabólica (Bull \& Dick, 1969; Cano-Gutierréz et al., 2012).

Megalocitose é alteração associada comumente a processos degenerativos do fígado ou a processos regenerativos dos hepatócitos (Bull \& Dick, 1960; Butler \& Barnes, 1963).

Megalocitose já foi descrita como uma lesão resultante de alterações potenciais em proteínas reguladoras do ciclo celular, incluindo alquilação de constituintes nucleares (Cano-Gutierréz et al., 2012) ou de aumento da atividade metabólica (Tokarnia, Döbereiner, Peixoto, Brito \& Barbosa, 2012). Megalocitose foi observada em pacientes submetidos a hepatectomia parcial, sendo neste caso, considerado um sinal de regeneração tecidual por parte dos hepatócitos (Zedeck \& Sternberg, 1975). Hipertrofia hepática é um achado comumente observado após a lesão hepática, entre outros fatores, devido a megalocitose regenerativa (Jago, 1969; Svoboda, Reddy \& Bunyaratvej, 1971).

As alterações qualitativas e quantitativas do tecido hepático verificadas neste estudo contradizem os resultados de Ben-Menachem (2004), porém corroboram as alterações bioquímicas encontradas em outros estudos (Einarsdottir \& Björnsson, 2008; Sendra, Junyent, \& Pellicer, 2011).

Embora tenha havido redução da densidade do volume do tecido intersticial na fase aguda do tratamento, o aumento do volume total do fígado implicou num aumento do volume absoluto do tecido intersticial.

Ocorreu aumento relativo e absoluto do volume do parênquima hepático aos 14 dias de vida, expressos pela densidade de volume e pelo volume absoluto dos componentes teciduais hepáticos respectivamente, indicando crescimento do órgão acima da média para os outros tecidos corpóreos.

Embora não tenha havido crescimento percentual de nenhum dos segmentos do órgão aos 21 dias de vida, houve crescimento absoluto do órgão expresso por meio do volume do fígado. Este fato influenciou os resultados obtidos para o parênquima e interstício hepáticos, o que nos sugere que o crescimento do fígado na fase inicial do tratamento foi maior do que 
na fase tardia, sobretudo, o crescimento do parênquima. No grupo tratado de 21 dias de vida houve aumento apenas do volume absoluto de ambos os componentes teciduais, revelando proporcionalidade no aumento desses elementos.

A megalocitose dos hepatócitos verificada aos 14 e 21 dias de tratamento possivelmente influenciou positivamente para o aumento de volume dos componentes teciduais hepáticos.

Em muitos processos patológicos, as alterações morfológicas precedem as alterações funcionais. Os resultados da bioquímica hepática encontrados nessa pesquisa são semelhantes aos descritos em outros estudos (Almeida Junior et al. 2020; Bonfim et al. 2021; Rodrigues et al. 2021; Rombi et al. 2021). Vários estudos tem avaliado a funcionalidade de hepatócitos megalocíticos, nos quais têm-se constatado que essas células continuam funcionalmente ativas durante o processo de hipertrofia, fazendo com que as alterações morfológicas não se reflitam, até este grau de comprometimento, em alterações funcionais expressas pelo aumento das enzimas hepáticas (Svoboda, Reddy \& Bunyaratvej, 1971).

\section{Conclusão}

A ingestão residual de pregabalina no leite materno provoca alterações estruturais nos hepatócitos dos ratos lactentes aos 14 e 21 dias de vida, sem alterações funcionais aparentes destas células e do fígado como um todo. Pesquisas futuras com um modelo experimental com tempo de lactação mais prolongado são necessárias, para analisar o impacto da ingestão residual crônica da droga em relação as alterações funcionais e morfológicas do hepatócito. É de bom alvitre que o laboratório fabricante reveja as informações contidas na bula do medicamento. É recomendável que pacientes em período de lactação que precisem fazer uso regular da droga ou em altas doses recorram ao banco de leite e colostro para fornecer alimento adequado a prole. Além disso, o acompanhamento periódico das enzimas hepáticas de pacientes que fazem uso crônico ou dose elevada de pregabalina pode contribuir sobremaneira para prevenção de alterações funcionais graves do fígado.

\section{Agradecimentos}

O trabalho foi realizado com apoio do CNPq, Conselho Nacional de Desenvolvimento Científico e Tecnológico Brasil, e desenvolvido junto ao Programa de Iniciação Científica da Universidade Federal de Campina Grande (PIBIC/CNPqUFCG).

\section{Referências}

Almeida Junior, S., Pereira, P. M., de Souza, Totoli, V., Carrijo, M. S., Badoco, F. R., de Freitas Pereira, L., \& Furtado, R. A. (2020). Validação de parâmetros do perfil renal e hepático de roedores Rattus novergicus mantidos no biotério de manutenção da Universidade de Franca. Research, Society and Development, 9 (5), e18952619-e18952619.

Arroyo, S., Anhut, H., Kugler, A. R., Lee, C. M., Knapp, L. E., Garofalo, E. A., Messmer, S., \& the Pregabalin 1008-011 International Study Group. (2004). Pregabalin Add-on Treatment: A Randomized, Double-blind, Placebo-controlled, Dose-Response Study in Adults with Partial Seizures. Epilepsia, 45(1), 2027.

Ben-Menachem, E. (2007). Pregabalin pharmacology and its relevance to clinical practice. Epilepsia, 45, 13-18. https://doi.org/10.1111/j.00139580.2004.455003.x.

Beydoun, A., Uthman, B. M., Kugler, A. R., Greiner, M. J., Knapp, L. E., Garofalo, E. A., \& Pregabalin 1008-009 Study Group. (2005). Safety and efficacy of two pregabalin regimens for add-on treatment of partial epilepsy. Neurology, 64(3), 475-80. https://doi.org/10.1212/01.WNL.0000150932.48688.BE.

Bonfim, D. J. P., Garcia, F. M., Laposy, C. B., Giuffrida, R., Nai, G. A., \& Bremer-Neto, H. (2021). Influence of water pH in the hepato-and nephrotoxicity of chronic cadmium poisoning in Wistar rats. Research, Society and Development, 10(9), e12210917753-e12210917753.

Brilhante, O. (1999). Estudo morfométrico e estereológico dos testículos de ratos albinos, imaturos sexualmente, tratados subcronicamente com doxorrubicina. Dissertação de Mestrado, Universidade Federal de São Paulo, São Paulo, SP, Brasil.

Bull, L. B., \& Dick, A. T. (1960). The function of total dose in the production of chronic lethal liver disease in rats by periodic injections of the pyrrolizidine alkaloid, heliotrine. Australian Journal of Experimental Biology and Medical Science, 38(6), 515-523.

Butler, W. H., \& Barnes, J. M. (1963). Toxic effects of groundnut meal containing aflatoxin to rats and guinea-pigs. British journal of cancer, 17(4), 699. 
Cabrera, J., Emir, B., Dills, D., Murphy, T. K., Whalen, E., \& Clair, A. (2012). Characterizing and understanding body weight patterns in patients treated with pregabalin. Curr Med Res Opin., 28(6), 1027-37. https://doi.org/10.1185/03007995.2012.684044.

Cano-Gutiérrez, G., Acevedo-Nava, S., Santamaría, A., Altamirano-Lozano, M., Cano-Rodríguez, M. C., \& Fortoul, T. I. (2011). Hepatic megalocytosis due to vanadium inhalation: participation of oxidative stress. Toxicol Ind Health, 28(4), 353-60. https://doi.org/10.1177/0748233711412424.

Cano-Paniagua, A., Amariles, P., Angulo, N., \& Restrepo-Garay, M. (2019). Epidemiology of drug-induced liver injury in a University Hospital from Colombia: updated RUCAM being used for prospective causality assessment. Annals of hepatology, 18(3), 501-507.

Cardia, G. F. E., de Souza Silva-Comar, F. M., Silva, E. L., da Rocha, E. M. T., Comar, J. F., Silva-Filho, S. E., ... \& Cuman, R. K. N. (2021). Lavender (Lavandula officinalis) essential oil prevents acetaminophen-induced hepatotoxicity by decreasing oxidative stress and inflammatory response. Research, Society and Development, 10(3), e43410313461-e43410313461.

Cunningham, J. G., \& Klein, B. G. (2008). Tratado de Fisiologia Veterinária (4ª Edição). Rio de Janeiro: Editora Elsevier Guanabara Koogan S.A.

Toledo, J. C., Toledo, C., DeCerce, J., \& Ramsay, R. E. (1997). Changes in body weight with chronic, high-dose gabapentin therapy. Ther Drug Monit.,19(4), 394-6. https://doi.org/10.1097/00007691-199708000-00006.

Einarsdottir, S, \& Björnsson, E. (2008) Pregabalin as a probable cause of acute liver injury. Eur J Gastroenterol Hepatol, 20(10), 1049. https://doi.org/10.1097/MEG.0b013e328300c978.

Gaspari, F., Cravedi, P., Mandalà, M., Perico, N., de Leon, F. R., Stucchi, N., Ferrari, S., Labianca, R., Remuzzi, G., \& Ruggenenti, P. (2010). Predicting Cisplatin-Induced Acute Kidney Injury by Urinary Neutrophil Gelatinase-Associated Lipocalin Excretion: A Pilot Prospective Case-Control Study. Nephron Clin Pract, 115, 154-160. https://doi.org/10.1159/000312879.

González-Escalada, J. R. (2005). Pregabalina en el tratamiento del dolor neuropático periférico. Revista de la Sociedad Española del Dolor, 12(3), 169-180.

Gunderson, D. R., \& Dygert, P. H. (1988). Reproductive effort as a predictor of natural mortality rate. ICES Journal of Marine Science, 44(2), 200-209. https://doi.org/10.1093/icesjms/44.2.200.

Jago, M. V. (1969). The development of the hepatic megalocytosis of chronic pyrrolizidine alkaloid poisoning. The American journal of pathology, 56(3), 405.

Jallon, P., \& Picard, F. (2001). Bodyweight Gain and Anticonvulsants. Drug-Safety, 24, 969-978. https://doi.org/10.2165/00002018-200124130-00004.

Kvamme, E., Torgner, I. A., \& Roberg, B. (2001). Kinetics and localization of brain phosphate activated glutaminase. Journal of neuroscience research, 66(5), 951-958. https://doi.org/10.1002 / jnr.10041.

Machado, A. R., da Silva Rodrigues, R., de Assis, L. M., Ribeiro, C. L. G., Rocha, A. D. S. R., Machado, M. R. G., \& de Souza-Soares, L. A. (2021). Estudos bioquímios e histopatológicos do fígado de ratos wistar alimentados com Spirulina cepa LEB-18 em tamanho micrométrico e nanométrico. Research, Society and Development, 10(1), e50310111654-e50310111654.

Mandarim-de-Lacerda, C. A. (1995). Métodos quantitativos em morfologia. Rio de Janeiro: EdUERJ.

Mandarim-de-Lacerda, C. A. (2003). Stereological tools in biomedical research. Anais da Academia Brasileira de Ciências, 74(4), 469-486. https://doi.org/10.1590/s0001-37652003000400006.

Martins, J. O., Panício, M. I., Dantas, M. P. S., \& Gomes, G. N. (2014). Efeito do diabetes materno na prole feminina. einstein (São Paulo), 12(4), 413-419. https://doi.org/10.1590/S1679-45082014AO3200.

Medeiros, J. P., dos Santos Silva, R. R., de Brito, L. P., da Costa, M. A. S., Falcão, D. M. S., da Silva, M. E., ... \& Neto, J. E. (2021). Aspectos toxicológicos da ivermectina: um estudo dos efeitos na morfologia do fígado de ratas prenhes. Research, Society and Development, 10(1), e25410111693-e25410111693.

Michalany, J. (1998). Técnica histológica em anatomia patológica: com instruções para o cirurgião, enfermeiras e citotécnico ( $3^{\circ}$ Ed). São Paulo: Michalany.

Miraglia, S. M.; \& Hayashi, H. (1993). Histomorphometry of immature rat testis after heating. Journal of morphology, 217(1), 65-74. https://doi.org/10.1002/jmor.1052170106.

Ness-Abramof, R., \& Apovian, C. M. (2005). Ganho de peso induzido por drogas. Drugs of Today, 41(8), 547-555. https://doi.org/10.1358 / dot.2005.41.8.893630.

Rodrigues, A. A., Alves, E. H. P., de Andrade, R. S. B., de Carvalho França, L. F., dos Santos Pessoa, L., da Silva, F. R. P., \& Vasconcelos, D. F. P. (2021). Modelo experimental de dieta rica em gordura é útil para avaliar o agravamento de danos ao fígado associados a comorbidades. Research, Society and Development, 10 (3), e1310313012-e1310313012.

Russell, L. D.; França, L. R. (1995). Building a testis. Tissue \& Cell, 27, 129-147. https://doi.org/10.1016/S0040-8166(95)80016-6

Scherle, W. (1970). A simple method for volumetry of organs in quantitative stereology. Mikroskopie, 26, 57-63.

Seawright, A. A., Hrdlicka, J., Wright, J. D., Kerr, D. R., Mattocks, A. R., \& Jukes, R. (1991). The identification of hepatotoxic pyrrolizidine alkaloid exposure in horses by the demonstration of sulphur-bound pyrrolic metabolites on their hemoglobin. Vet Hum Toxicol, 33(3), 286-7.

Sendra, J. M., Junyent, T. T., \& Pellicer, M. J. R. (2011). Pregabalin-Induced Hepatotoxicity. Annals of Pharmacotherapy, 45(6), e32-e32. https://doi.org/10.1345/aph.1Q032.

Siddall, P. J., Cousins, M. J., Otte, A., Griesing, T., Chambers, R., \& Murphy, T. K. (2006). Pregabalin in central neuropathic pain associated with spinal cord injury: a placebo-controlled trial. Neurology, 67, 1792-800. 
Research, Society and Development, v. 10, n. 16, e491101624117, 2021

(CC BY 4.0) | ISSN 2525-3409 | DOI: http://dx.doi.org/10.33448/rsd-v10i16.24117

Silveira, I. D., Roos, D. H., Salgueiro, A. C. F., Folmer, V., da Rocha, J. B. T., \& Puntel, R. L. (2020). Haloperidol aumenta a peroxidação lipídica hepática promovida por uma dieta rica em gordura em ratos Haloperidol increases hepatic lipid peroxidation promoted by high-fat diet in rats El haloperidol aumenta la lipoperoxidación en tejido hepático promocionada por una dieta alta en grasas en ratas. Research, Society and Development, 9(2), e148922153.

Svoboda, D., Reddy, J., \& Bunyaratvej, S. (1971) Megalocitose hepática em intoxicação crônica por lasiocarpina: Alguns estudos funcionais. The American Journal of Pathology, 65(2), 399-409.

Taylor, C. P., Angelotti, T., \& Fauman, E. (2007). Pharmacology and mechanism of action of pregabalin: the calcium channel $\alpha 2-\delta$ (alpha2-delta) subunit as a target for antiepileptic drug discovery. Epilepsy research, 73(2), 137-150. https://doi.org/10.1016/j.eplepsyres.2006.09.008.

Tokarnia, C. H., Döbereiner, J., Peixoto, P. V., Brito, M. F., \& Barbosa, J. D. (2012). Plantas tóxicas do Brasil para animais de produção. Rio de janeiro: Helianthus.

Weibel, E. R. (1981). Stereological methods. Vol. 1: Practical methods for biological morphometry. Journal of Microscopy, 121(1), 131-132. https://doi.org/10.1111/j.1365-2818.1981.tb01205.x

Zedeck, M. S., \& Sternberg, S. S. (1975). Megalocitose e outras anormalidades expressas durante a proliferação no fígado em regeneração de ratos tratados com acetato de metilazoximetanol antes da hepatectomia parcial. Pesquisa do câncer, 35(8), 2117-2122. 